\title{
Local Surface Orientation Dominates Haptic Curvature Discrimination
}

\author{
Maarten W. A. Wijntjes, Akihiro Sato, Vincent Hayward, Fellow, IEEE, and Astrid M. L. Kappers
}

\begin{abstract}
Prior studies have shown that local surface orientation is a dominant source of information for haptic curvature perception in static conditions. We show that this dominance holds for dynamic touch, just as was shown earlier for static touch. Using an apparatus specifically developed for this purpose, we tested this hypothesis by providing observers with two independently controlled sources of geometric information. The robotic-like apparatus could accurately control the position of a contact surface independently from its orientation in space, while allowing subjects to freely and actively explore virtual shapes in the lateral direction. In the first experiment, we measured discrimination thresholds for the two types of shape information and compared the discrimination of real shapes to that of virtual shapes. The results confirmed the dominance of local surface orientation. We propose a model that predicts cue dominance for different scales of exploration. In the second experiment we investigated whether a virtual curved surface felt as curved as a real curved surface. We found that observers did not systematically judge either of the two kinds of stimuli to be more curved than the other. More importantly, we found that points of subjective curvedness were not influenced by the availability of height information.
\end{abstract}

Index Terms-Haptic Curvature Perception, Haptic Devices, Real and Virtual Shapes.

\section{INTRODUCTION}

$\mathbf{T}$ OUCH, like vision or audition, is thought to operate from multiple sources of information, or 'cues', that relate the attributes of an object such as its size, shape, or weight, to the sensing and computational processes employed to perceive them. By similarity with other senses, it should be possible to precisely describe the haptic cues which, along with prior information not accessible to the senses, allow the brain to recover objects attributes [1]. In this paper, we look at the curvature discrimination of stationary, rigid objects. With an apparatus that allowed us to manipulate cues related to the geometry of profiles, we found that subjects relied on surface orientation almost exclusively to perceive differences in curvature smaller than $\pm 2 \mathrm{~m}^{-1}$ and when the span of finger exploration is $18 \mathrm{~cm}$. Further, we developed a model that predicts the ranges within which different sources of information dominate in the perception of haptic shape, considering position, slope, and surface curvature. The model draws from our own data and that of a number of results found in the literature.

M. W. A. Wijntjes and A. M. L. Kappers are with the Department of the Physics of Man, Helmholtz Institute, Utrecht University, the Netherlands. Email: \{M.W.A.Wijntjes|A.M.L.Kappers\}@uu.nl

A. Sato and V. Hayward are with the Haptics Laboratory, McGill University, Montreal, Quebec, Canada. E-mail: \{aki|hayward\}@cim.mcgill.ca Manuscript received June 2008.

\section{A. Preliminaries}

A two-dimensional shape, such as the profile of an extruded solid, may be specified by the locus of a point, $\boldsymbol{p}(s)$ that describes a curve parametrized by a path length $s$ (see Fig. 1). This specification is a general description of a two-dimensional shape. A smooth curve defines a tangent vector $\boldsymbol{t}=\mathrm{d} \boldsymbol{p}(s) / \mathrm{d} s$ at all points. Given $\varphi(s)$, the angle that the vector $\boldsymbol{t}$ makes with the horizontal direction, a signed curvature can be defined as $c(s)=\mathrm{d} \varphi(s) / \mathrm{d} s$. A normal vector $\boldsymbol{n}(s)=\mathrm{d} \boldsymbol{t}(s) / \mathrm{d} \varphi$ orthogonal to $\boldsymbol{t}(s)$ is therefore determined. Given appropriate integration constants, by means of the Frenet formulas $\mathrm{d} \boldsymbol{t}(s) / \mathrm{d} s=c \boldsymbol{n}(s)$ and $\mathrm{d} \boldsymbol{n}(s) / \mathrm{d} s=-c \boldsymbol{t}(s)$, these quantities are related to each other through transformations involving differentiation and integration. A circular profile, in particular, has a constant curvature, $c=1 / r$, that is the inverse of its radius $r$. It can be readily verified that integrating a constant curvature along the path $s$ twice, gives the equation of a circle.

The position, $\boldsymbol{p}(s)$, slope, $\varphi(s)$, and curvature, $c(s)$, are shape descriptors which are presumably all available to the human haptic channel as illustrated in Fig. 1. When a finger explores a shape, the profile determines the location $\boldsymbol{p}(s)$, the orientation of the surface $\boldsymbol{n}(s)$, and the local curvature $c(s)$. Each of these quantities is accessible through touch, although complicated transformations may be necessary to transform the raw sensory input into representations that can be used by the brain. The position, the orientation and the curvature of a small contact surface are termed zeroth, first and second order shape descriptors, respectively.

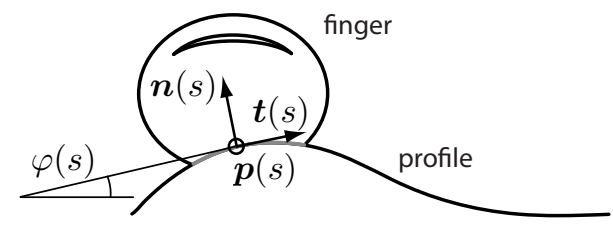

Fig. 1. Geometric information available for an exploring finger. The position, $\boldsymbol{p}(s)$ (zeroth order), the orientation of the normal vector, $\boldsymbol{n}(s)$ (first-order), and the local curvature $c(s)$ (second order, gray curve) are related to each other.

\section{B. Previous Work}

Several previous studies have suggested that the orientation of the surface of contact is an important source of information for curvature perception. Gordon and Morison asked observers to explore $2 \mathrm{~cm}$ long, curved profiles and found the $75 \%$ discrimination threshold is approximately $2 \mathrm{~m}^{-1}$ [2]. Pont et al. performed a similar experiment but used longer, $20 \mathrm{~cm}$ 
profiles and found a $84 \%$ discrimination threshold to be about $0.5 \mathrm{~m}^{-1}$ [3]. In fact, Gordon and Morison had suggested that the threshold dependency on exploration path length could be explained, not by the curvature directly, but rather by the total variation of the orientation of the surface. In our terminology, the stimulus can be said to be the integral of the curvature (i.e. the total slope change), which makes the dependency on path length evident. This idea was further developed by Pont et al. who showed that for static touch, the total orientation variation, the first-order shape descriptor, was also the effective stimulus [3]. In the same study, evidence supporting the notion that a similar mechanism underlies static and dynamic touch was supplied by varying the exploration path length. The discrimination thresholds consistently increased with decreasing path lengths, but in terms of orientation differences, the thresholds remained the same.

These findings say nothing about the relative contributions of zeroth and first-order shape for dynamic touch since these two aspects of geometry were always correlated in the physical stimuli employed in these studies. Measuring discrimination thresholds for each source requires to deliver zeroth and first-order geometric information independently. A technique that can accomplish segregated cue delivery was proposed by Dostmohamed and Hayward who designed a device that could render a shape using first-order information only [4]. The fingertip was placed on a surface which could be freely moved horizontally. Its orientation was controlled such that it would replicate the orientation of surfaces. They found that the simplified stimulus induced curvature perception, even in the absence of zeroth and second order geometric information.

\section{Aims Of The Study}

Our study serves two goals. The first is to gain knowledge regarding the specific contributions of geometrical cues in the haptic perception of shape. By contrast, it is known that haptic shape perception also depends on non-geometric features. For instance, the force field arising from a haptic interaction can induce the perception of illusory shapes independently from the geometrical information given by a rigid profile [5]. It was further shown that humans perceptually combine force field cues with zeroth order shape information [6]. From this viewpoint, the result of our study can form the basis for further studies in intra-modal cue integration where zeroth-, first-, and second-order information and their correlates would be segregated and re-combined in various ways.

The second goal is to inform designers of haptic interfaces of the methods that are available to render shapes haptically. If for certain applications aspects of the geometric information can be discarded then the resulting system would be simpler and more effective. Conversely, the results of experiments performed with devices that rely on zeroth order information should be discussed in the light of the present findings. For example, Henriques and Soechting investigated haptic perception using a manipulandum [7]. Their findings, however, might not generalize to geometrically richer stimuli. By the same token, our results easily explain the poor performance of traditional haptic interfaces when users must deal with low curvature shapes [8], and suggest new directions for the design of haptic interfaces [9].

\section{Approach}

Here, we used a device similar to that used in [4], but improved the design such that also the position, i.e. zeroth order geometric information, could be varied independently from orientation, i.e., first-order geometric information. Referring to Fig. 2, the apparatus' function is to allow a user to explore a surface simulated by a surface moving freely in the horizontal $x$-direction. The displacement, $x$, in the horizontal direction is measured and is used to determine under servo control the elevation, $z$, and the angle, $\varphi$, the surface makes with the horizontal direction.

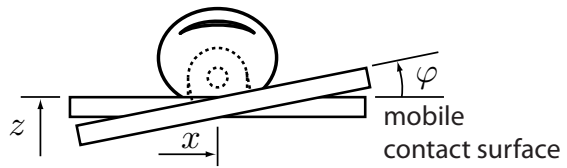

Fig. 2. Degrees of freedom of the contact surface. Movement in the horizontal $x$-direction is free but the elevation, $z$, and the angle, $\varphi$, are servo-controlled. See reference [10] for details.

With this apparatus, many types of stimuli can be generated by combining arbitrary combinations of zeroth and first-order geometric information. This is illustrated in Fig. 3(a)-(c). By holding certain aspects of the stimulus constant during exploration, namely elevation for zeroth-order information, orientation for first-order information, the corresponding cues become uninformative. In addition, since the finger is always in contact with a flat surface, cues arising from second-order information are made inoperative.

Goodwin et al. found that observers using a single static finger could not discriminate a flat surface from a curved one (at a $84 \%$ level) when the curvature was smaller than $7.5 \mathrm{~m}^{-1}$ [11]. The curvatures that we used were all well below this value. Statically, they could not be distinguished from a flat surface. If observers used geometric information only, the virtual and real stimuli, Fig. 3(c),(d), would be equally well discriminated. However, there certainly exist nongeometric differences between real and rendered shapes that result from the differences in the mechanical interactions between conditions which may be of influence. To account for the roles of non-geometric cues, we included real, solid stimuli in our investigation.

In two experiments we tested the role of zeroth and firstorder shape information on haptic perception. In the first experiment we measured discrimination thresholds as the just noticeable differences, or JNDs, for three combinations of shape descriptors as well as for real shapes. We used a flat reference and were thus measuring "just noticeable curvedness." In the second experiment we wanted to understand whether a virtual shape felt equally curved as a real shape. To investigate this question, we tested for the existence of a perceptual bias between real and virtual shapes. Our specific goal was to test whether a shape rendered only by the first-order shape descriptor, i.e. the orientation of the contact area surface, gives 
(a)

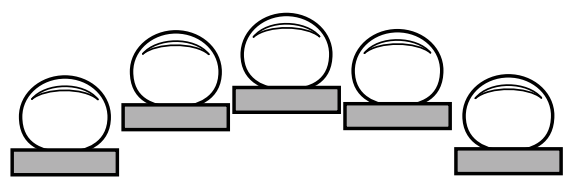

(b)

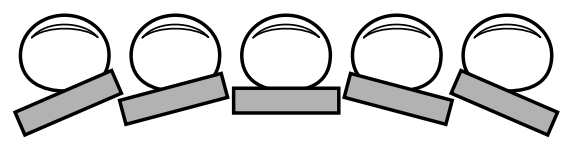

(c)

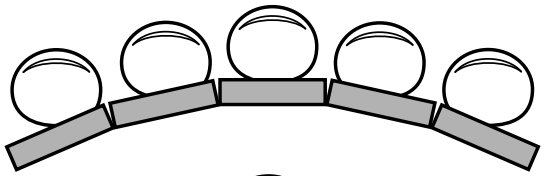

(d)

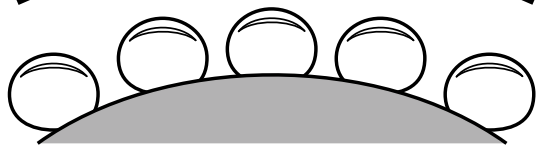

(e)

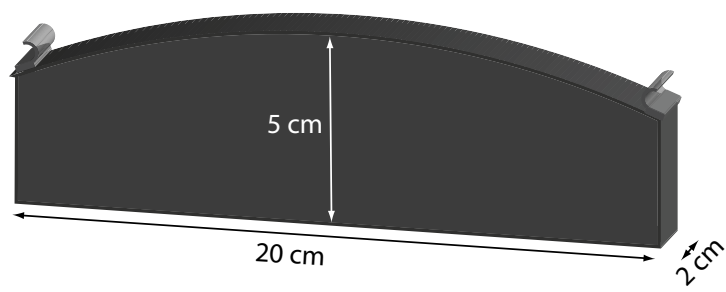

Fig. 3. The four conditions used in Experiment 1. The observer experiences curvature through (a) zeroth-order geometric information only, (b) first-order geometric information. In (c), the two sources are combined. The fourth condition, (d), is an actual solid. Its geometry is shown in panel (e). Panels (a)-(c) illustrate the movements of a finger and its relation with the contact surface with five frames, the actual movement, however, was continuous. The arrow indicates one full cycle of finger motion.

a bias similar to that given by the cue combination in which height information is also present. In the present study all stimuli were truncated circular profiles, i.e. having constant curvature.

\section{EXPERIMENT 1}

\section{A. Methods}

1) Participants: Eight observers participated in the first experiment. They were recruited from the McGill campus and were reimbursed for their participation. None of the observers had previously participated in a related study and they were naive as to the purpose of the study. The group consisted of four males and four females, with a mean age ( \pm standard deviation) of $22.9 \pm 5.4$ years. According to Coren's handedness test, all observers were right-handed [12].

2) Stimuli: The device that could independently orient and elevate a moving contact surface is shown in Fig. 4. By placing the fingertip on the surface, an observer could move the surface laterally. The orientation and elevation depended on the contact position according to the profile to be represented. Despite careful engineering (low friction guidance, lightened mechanism), it was unavoidable that observers had to displace a non zero mass during shape exploration. However, the results did not indicate any systematic differences that could be attributed to inertia or friction and which could cause differences in perception between the real and virtual shapes. A detailed description of the device and information about the performance can be found in [10].

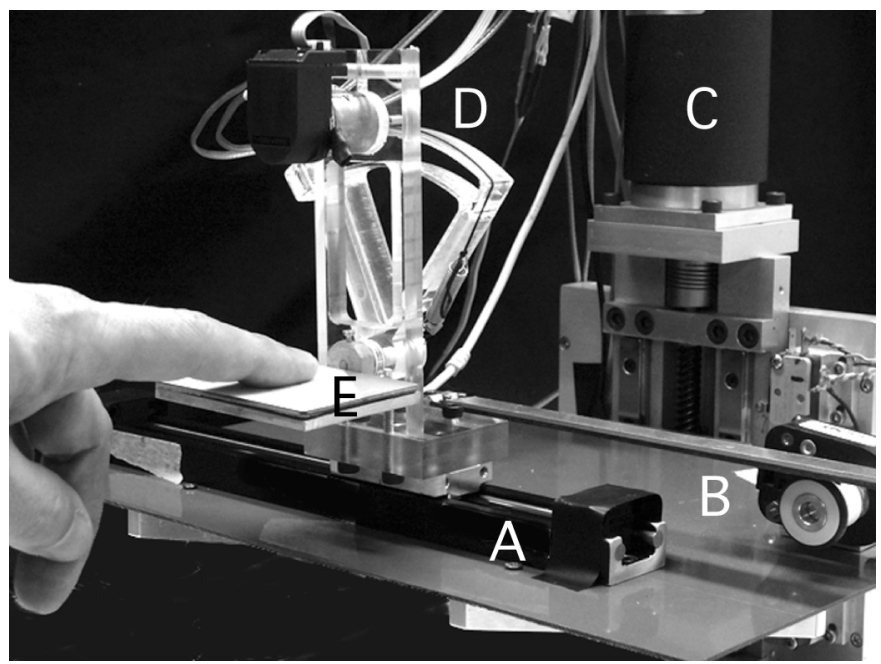

Fig. 4. Apparatus to produce shape stimuli with segregated cues. During exploration, the finger moved the contact surface $E$ laterally guided by a low friction linear bearing $A$. The encoder $B$ measured the horizontal displacement used to compute a shape represented by height and/or slope. The servo mechanism $C$ controlled elevation and servo mechanism $D$ the slope.

The real stimuli were curved strips made from PVC plastic, see Fig 3(e). They were $20 \mathrm{~cm}$ long, $2 \mathrm{~cm}$ wide and at the midpoint had a height of $5 \mathrm{~cm}$, regardless of their curvature [3]. Cylindrical stops (height $7 \mathrm{~mm}$ ) at each end of the upper surface determined a movement range of $18 \mathrm{~cm}$ without giving unwanted shape cues as described in reference [3].

Referring to Fig. 3, condition (a) had zeroth order shape information only, the plate remained horizontal. Condition (b) had first-order shape information only. The height of the plate remained constant. Condition (c) had both zeroth and firstorder shape information. In condition (d), the real stimuli were used, providing 0th, 1st and 2nd order information.

3) Procedure: Despite precautions in testing and tuning the device, see [10], we measured JND's using a paradigm that eliminated the effects of possibly uncontrolled cues. Such unwanted cues can include minute vibrations, small enough to be under conscious threshold, yet picked up by the tactile or auditory systems and used to detect non-flatness. We used a two-alternative-forced-choice task. In each trial, observers had to judge which of two stimuli was "more convex" where one had a zero reference curvature and the other a non-zero test curvature. The order of test and reference stimuli was counterbalanced and randomized. Therefore, any bias due to unwanted cues would cancel out in the limit since observers had to indicate the sign of the curvature difference between stimuli.

The four conditions were tested separately in a blocked design. The order of the conditions was semi-counterbalanced since the number of permutations of conditions exceeded the number of participants. We ensured that the real shape condition was balanced among the virtual shape conditions. Before the experiment the experimenter verified that the participants 
had a clear notion of what a convex shape meant. During the experiment, a depiction of a convex shape was posted in view of the subjects.

The participants wore sound isolating headphones and were seated behind a curtain that concealed the apparatus. They were not permitted to see it until the experiment was completed. They were informed that during three of the four conditions they would feel a virtual shape but were not informed about the details of these three virtual conditions. The participants familiarized themselves with the task and received feedback on their answers for ten trials.

To determine the optimal samplings of the psychometric curves, each session had an initial block of 40 trials where each stimulus in the set of curvatures $\{-2,-1.8, \ldots,-0.2,0.2, \ldots, 1.8,2\} \mathrm{m}^{-1}$ was presented twice. From the resulting data an estimate of the individual thresholds could be found. The session continued with a stimulus set of 12 different test values that were chosen to bracket the estimated threshold. The resulting range was always limited by -4 and $4 \mathrm{~m}^{-1}$. The total number of repetitions per test stimulus was 10, making the total number of responses per discrimination threshold equal to 120 .

During the experiment all stimuli were located such that their midpoints were always at the same height. The real stimuli were placed in a jig. The participants were instructed to begin exploration at the midpoint of the stimuli; they made two full sweeps and moved back to the midpoint. Within a trial, each stimulus was experienced once. The participants had to decide which of the two stimuli was more convex and did not receive feedback on their responses. In the virtual conditions, the finger was positioned on the contact surface and in the real conditions the finger was positioned on the surface of the stimulus.

4) Data analysis and design: For each test-reference pair, the responses were transformed into fractions of 'test curvature feels more convex'. These values were fitted to a cumulative Gaussian function

$$
f(c)_{\sigma, \mu}=\frac{1}{\sigma \sqrt{2 \pi}} \int_{-\infty}^{c} e^{-\frac{\left(c^{\prime}-\mu\right)^{2}}{2 \sigma^{2}}} d c^{\prime}
$$

where $f(c)$ models the mean response for a test curvature $c$. The parameter $\sigma$ corresponds to the $84 \%$ discrimination threshold. The parameter $\mu$ was set to zero in the first experiment. In the second experiment, where stimuli were pairs from different conditions, $\mu$ corresponds to the Point of Subjective Equality (PSE). A maximum-likelihood procedure was used to fit the function as described by [13]. The discrimination thresholds resulting from the fit procedure were analysed in a one-way repeated measures Analysis of Variance (ANOVA) of the four conditions depicted in Fig. 3.

\section{B. Results}

The individual and mean results are collected in Fig. 5. The threshold of participant 1 for the height-only condition was outside the stimulus range. The data from this participant was thus unreliable and could not be included in the statistical analyses. The mean threshold for the height-only condition was about four times greater than in the other conditions. A significant main effect was confirmed by Greenhouse-Geiser corrected ANOVA $(F(1.036)=15.239, p=0.005)$. Bonferroni corrected pairwise comparisons only revealed significant differences $(p<0.05)$ between the height-only condition and the other three conditions.

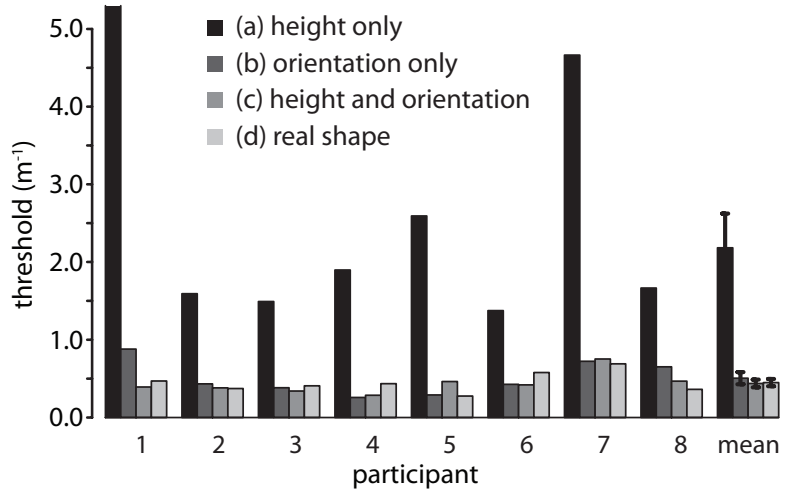

Fig. 5. Results of Experiment 1. The thresholds are shown for each participant in the four experimental conditions. For the calculation of the mean results, the threshold of participant 1 in condition "height only" was omitted. Error bars denote standard errors of the means.

\section{Discussion}

The results demonstrate that discrimination performance depends largely on the availability of orientation information. A curved shape that is defined solely by a height profile is hard to discriminate. Moreover, there is no apparent difference between virtual and real stimuli. Once orientation information is available in a real or a virtual stimulus, curvature discrimination performance becomes similar.

The thresholds for zeroth and first-order shapes with dynamic touch are similar to those found in reference [3] for static touch. The change of orientation of the contact surface seems to be the effective stimulus for curvature discrimination in static as well as in dynamic touch. However, finding similar discrimination thresholds does not demonstrate that a shape rendered solely by orientation information actually feels curved. Such demonstration would be an important step towards understanding haptic shape perception. Therefore, we conducted a second experiment.

\section{EXPERIMENT 2}

In this experiment we investigated whether an orientationonly shape is actually perceived as being curved. A secondary aim was to investigate whether a shape of which both the height and the orientation are rendered feels equally curved as a real shape. To answer these questions we compared shapes rendered in conditions (b) and (c) to real shapes (d). Observers determined the points of subjective equality (PSE) between real and rendered shapes using the method of constant stimuli. To ensure that height information was perceivable, we used a reference curvature of $2 \mathrm{~m}^{-1}$ which corresponds to the mean $84 \%$ correct threshold level found in the previous experiment (see the black bars in Fig. 5). We also hypothesized that in Experiment 2, the thresholds would be higher than those found in Experiment 1 due to the direct comparison of real and synthesized stimuli. 


\section{A. Methods}

1) Participants: For the second experiment we recruited a new group of eight participants from the McGill campus (three males and five females, age $24.2 \pm 5.0$ year). They were naive with respect to the purpose of the study and did not participate in other studies on haptic curvature perception. They were reimbursed for their participation. According to Coren's handedness test all participants were right-handed [12].

2) Stimuli: The stimuli were similar to those used in the first experiment, however, they were now all convex. The reference curvature was $2 \mathrm{~m}^{-1}$. The synthesized stimuli were presented in conditions shown in Figs. 3(b) and 3(c).

3) Procedure: A curtain concealed the apparatus from the seated participants. In each trial, they were presented with a pair of stimulus, one was a real shape and the other was synthesized. The participants had to decide which of the two stimuli felt more convex. When investigating points of subjective equality one must ensure that the only varying experimental condition is that under investigation. Since the two stimuli could not be presented in the exact same location, the space between them was minimized to $4 \mathrm{~cm}$. The real shapes were always positioned in a jig in front of the device. Participants were instructed to explore the stimulus 'in the front' (the real shape) or 'in the back' (the device, real stimulus removed). The only difference between the real and rendered stimuli was that location differed by $4 \mathrm{~cm}$ in the sagittal direction.

The two types of synthesized stimuli, with and without height information, were the two experimental conditions. These two conditions were measured in blocks and counterbalanced within the observer group. Within a block, the participants were always presented with a reference stimulus of $2 \mathrm{~m}^{-1}$ and a test stimulus. When the reference was a real shape, the test was a synthesized shape and vice versa.

Once the real shape was removed or put into place, participants could feel the second stimulus. Instructions regarding exploration were identical to those of the first experiment and participants had to judge which stimulus felt more curved by responding 'front' or 'back'. Before the experiment started, the experimenter made sure that the observers understood what was meant by 'more convex'. Participants could always consult a graphical illustration meant to clarify the task.

Before each block, participants were given 10 practice trials, and since we were interested in the perceptual bias between two different conditions, what was a correct answer was not defined. Therefore, no feedback was given, neither during training nor during the experiment. Moreover, the participants were not informed about the fact that differences existed regarding the availability of height information between the two conditions. For each condition the test curvatures were $2 \mathrm{~m}^{-1} \pm\{0,0.2,0.4,0.6,1.0,1.4\}$. Each test-reference pair was measured 12 times of which the real and rendered shapes each served six times as reference curvature. Thus, each block consisted of 132 trials.

4) Data analysis: The 'front-back' responses were converted into 'real shape feels more convex than the rendered shape' as a function of the curvature difference between the real and rendered shape, that is, $c_{\text {dif }}=c_{\text {real }}-c_{\text {virtual }}$. The perceptual bias $\mu$ is defined to be the curvature difference $c_{\mathrm{dif}}$ at which the psychometric function equals 0.5 . If real and virtual shapes would feel equally curved, there would be no bias, i.e. $\mu=0 \mathrm{~m}^{-1}$. If an orientation-only shape felt flat compared to a real shape, the bias would be $\mu=-2 \mathrm{~m}^{-1}$. To extract the bias from the data we used the same fitting procedure as in the first experiment, based on equation (1).

\section{B. Results}

Figure 6 shows the individual discrimination thresholds (JNDS) and biases (PSES). To test whether there was a significant difference in discrimination performance between Experiments 1 and 2, we conducted an unpaired $t$-test over the pooled data $(N=24)$ of conditions (b), (c) and (d) from Experiment 1 (which were not significantly different from each other) against the pooled data $(N=15)$ of Experiment 2 . This showed that discrimination was significantly better in Experiment $1\left(\sigma_{\text {mean }}=0.46 \mathrm{~m}^{-1}\right)$ than in Experiment 2 $\left(\sigma_{\text {mean }}=0.79 \mathrm{~m}^{-1}\right): t_{37}=-3.35, p<0.005$.
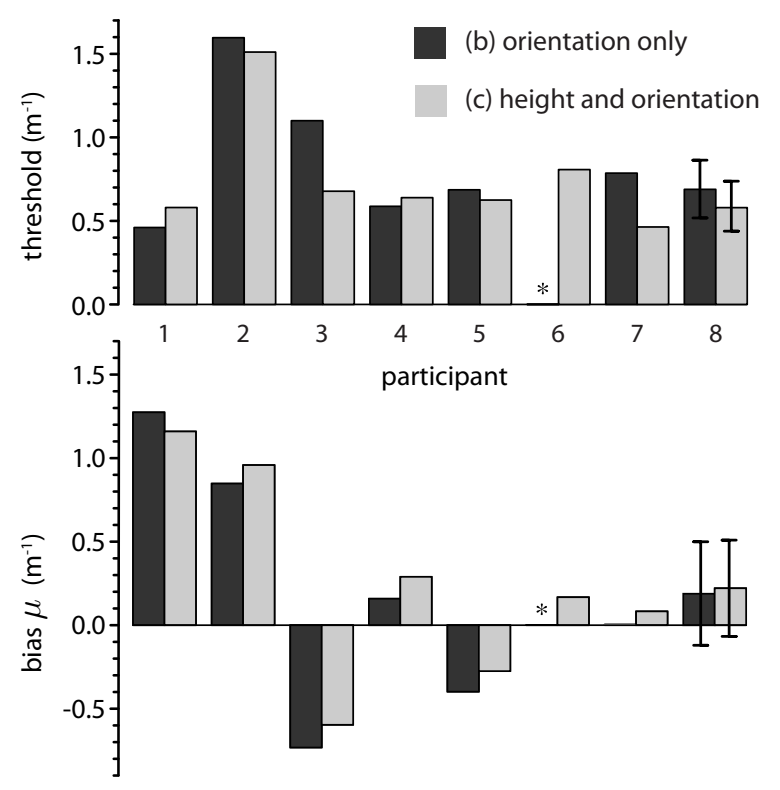

Fig. 6. Discrimination thresholds (JNDs) per condition for each participant (top panel). Biases (PSEs) per condition for each participant (bottom panel). Dark grey bars: height and orientation were available. Light grey bars: orientation only. The $*$-sign indicates omitted data, see text for details. For the mean results the fifth and sixth participant were omitted. Error bars denote standard errors.

The data set of participant 6 for the condition (b) in Experiment 2 was omitted because the threshold fell outside the measurement range and thus was not reliable. Participant 7 was accidentally measured twice in the condition (b) and not in (c), which was impossible to recover. Because the data are nevertheless of interest they are presented together with the data of the other participants in Fig. 6. The directions of the biases are not systematic but are consistent within participants across conditions. The differences between conditions were equally balanced.

Half of the participants (excluding participants 6 and 7) showed positive biases for both conditions and the other half showed negative biases. Consistency was further supported by 
a high correlation between the two conditions $\left(r^{2}=0.99\right.$, $p<0.0002)$. It is also important to note that all biases were well above the $-2 \mathrm{~m}^{-1}$ level. Such a bias would indicate that a virtual shape with a curvature of $2 \mathrm{~m}^{-1}$ has the same phenomenological curvature as a flat surface. The bias in question would be observed if observers relied only on zeroth order information when comparing a real shape with a shape represented by orientation only.

\section{Discussion}

The results show that adding zeroth-order shape information does not influence the perception of subjective curvature. Irrespectively of the condition, the observers did not systematically judge a rendered shape to have higher or lower curvature than a real shape. In addition, we found that the thresholds found in Experiment 2 were significantly higher than those found in Experiment 1. In Experiment 2 observers had to compare two different stimuli (synthesized vs. real) but in Experiment 1 the comparison was always performed between two similar stimuli. As hypothesized at the outset of this section, it is harder to compare real shapes with virtual shapes, than shapes experienced under the same conditions.

\section{General Discussion}

We can conclude that first-order shape information dominates haptic perception in the discrimination of curvature. This finding agrees with those of the study of Pont et al. who proposed that the slope difference is the effective stimulus of a curved surface [3]. Our results also confirm and extend the findings of Dostmohamed and Hayward to the effect that orientation information alone is sufficient to elicit a percept of curvature [4].

Intuitively, one should find it increasingly difficult to appreciate the curvature of a given surface from a change of height as the exploration movement becomes smaller. Conversely, consider a family of surface patches having the same slopes at each end. As they become wider, they also become higher. Similar observations can be made of the total change of slope experienced during exploration. Assuming that the human haptic perceptual system is sensitive to all three geometric sources of information discussed so far, that is, position, slope, and curvature of a contact surface between a finger and a rigid object, then it should be possible to predict the scales at which these sources of information are operative. Such a model is developed next.

\section{A. Relationships Between Perceptual Thresholds}

The truncated circular profiles used in our experiment are entirely determined by two parameters, a curvature and a exploration horizontal distance for example. For a given profile, see Fig. 7, these parameters may be expressed as combinations of the four quantities that seem to be perceptually relevant, namely, the curvature, $c$, the total change of height, $h$, the total orientation change, $\phi$, and the horizontal path length, $d$.

Mathematically, these quantities may be viewed as redundant coordinates of the stimulus space. Given any two of these

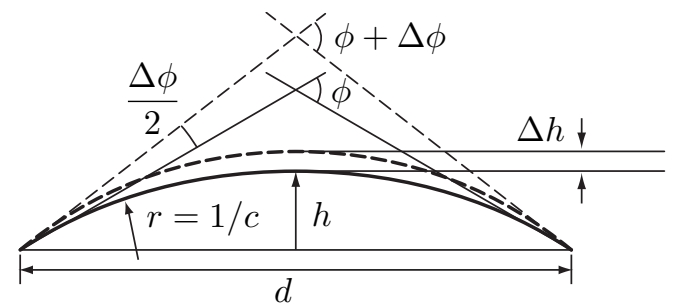

Fig. 7. Perceptual parametrization of a circular profile. Mathematically, only two parameters suffice but perceptually four matter.

TABLE I

RELATING THE FOUR PARAMETERS TO EACH OTHER.

$$
\begin{aligned}
h & =\frac{d}{2} \frac{1-\cos (\phi / 2)}{\sin (\phi / 2)} & h & =\frac{1}{c}-\sqrt{\frac{1}{c^{2}}-\frac{d^{2}}{4}} \\
\phi & =2 \sin ^{-1}\left(\frac{4 h d}{d^{2}+4 h^{2}}\right) & \phi & =2 \sin ^{-1}\left(\frac{d c}{2}\right) \\
c & =\frac{8 h}{d^{2}+4 h^{2}} & c & =\frac{2}{d} \sin (\phi / 2) \\
d & =2 h \frac{\sin (\phi / 2)}{1-\cos (\phi / 2)} & d & =\frac{2 \sin (\phi / 2)}{c}
\end{aligned}
$$

quantities, the other two can be found as indicated in Table I.

Further, any small change (as illustrated in Fig. 7) of any of these quantities, to first order approximation, can be related to a small change of any of the other three. In particular, given a horizontal movement path length $d$ we can express a threshold expressed with one quantity in terms of thresholds expressed with the other two,

$$
\begin{aligned}
& \Delta h(d)\left.\left.\simeq \frac{\partial h(d, \phi)}{\partial \phi}\right|_{\phi=0} \Delta \phi \simeq \frac{\partial h(d, c)}{\partial c}\right|_{c=0} \Delta c, \\
& \Delta \phi(d)\left.\left.\simeq \frac{\partial \phi(d, h)}{\partial h}\right|_{h=0} \Delta h \simeq \frac{\partial \phi(d, c)}{\partial c}\right|_{c=0} \Delta c, \\
&\left.\left.\Delta c(d) \simeq \frac{\partial c(d, h)}{\partial h}\right|_{h=0} \Delta h \simeq \frac{\partial c(d, \phi)}{\partial \phi}\right|_{\phi=0} \Delta \phi .
\end{aligned}
$$

The derivatives are evaluated for $c=\phi=h=0$ because we used a flat reference curvature. Using these relations, a threshold can be expressed using any of the quantities, $c, \phi$, or $h$ for a given exploration range $d$. Table II collects all the single-cue thresholds expressed with different quantities.

TABLE II

THRESHOLDS EXPRESSED IN DIFFERENT QUANTITIES FOR $d=18 \mathrm{CM}$. BOLD TYPEFACE VALUES REPRESENT THE MODEL PARAMETERS.

\begin{tabular}{lllll}
\hline Source & Threshold & $h(\mathrm{~mm})$ & $\phi\left({ }^{\circ}\right)$ & $c\left(\mathrm{~m}^{-1}\right)$ \\
\hline Exp 1A & $0^{\text {th }}$ & $\mathbf{8 . 8}$ & 22.5 & 2.2 \\
Exp 1B & $1^{\text {st }}$ & 2.1 & $\mathbf{5 . 3}$ & 0.5 \\
Goodwin et al. [11] & $2^{\text {nd }}$ & 30.4 & 77.3 & $\mathbf{7 . 5}$ \\
\hline
\end{tabular}

We can relate these observations to the results of Pont et al. who measured curvature discrimination thresholds, $\Delta c$, for various exploration path lengths between 5 and $20 \mathrm{~cm}$ [3]. They plotted the thresholds expressed with different quantities and found that the threshold expressed in orientation difference, $\Delta \phi$, was invariant with respect to the exploration path 
length, $d$. This finding led them to conclude that orientation difference is the effective source of information for curvature. However, their model does not account for the case in which the finger is not moving. It is known that second order shape information is statically sensed as shown by Goodwin et al. [11]. We conclude from these and our results that a more general model can be developed, accounting for the scale of exploration that can simply be expressed by the quantity $d$.

\section{B. A More General Model}

To generalize this model, we propose that the information derived from the three shape descriptors varies in dominance across the movement range scales. The construction of this model is illustrated in Fig. 8(a). The rows represent the variations of threshold changes for each shape descriptor expressed in terms of the other two when the scale $d$ varies. The discrimination threshold of a zeroth-order shape descriptor does not depend on $d$ when it is expressed as change in height. Similarly the threshold of a first and second-order shape description does not depend on $d$, when expressed as changes of angles or of curvature (see bold typeface values in Table II). The columns collect the individual thresholds expressed in terms of each quantity, using (2)-(4).

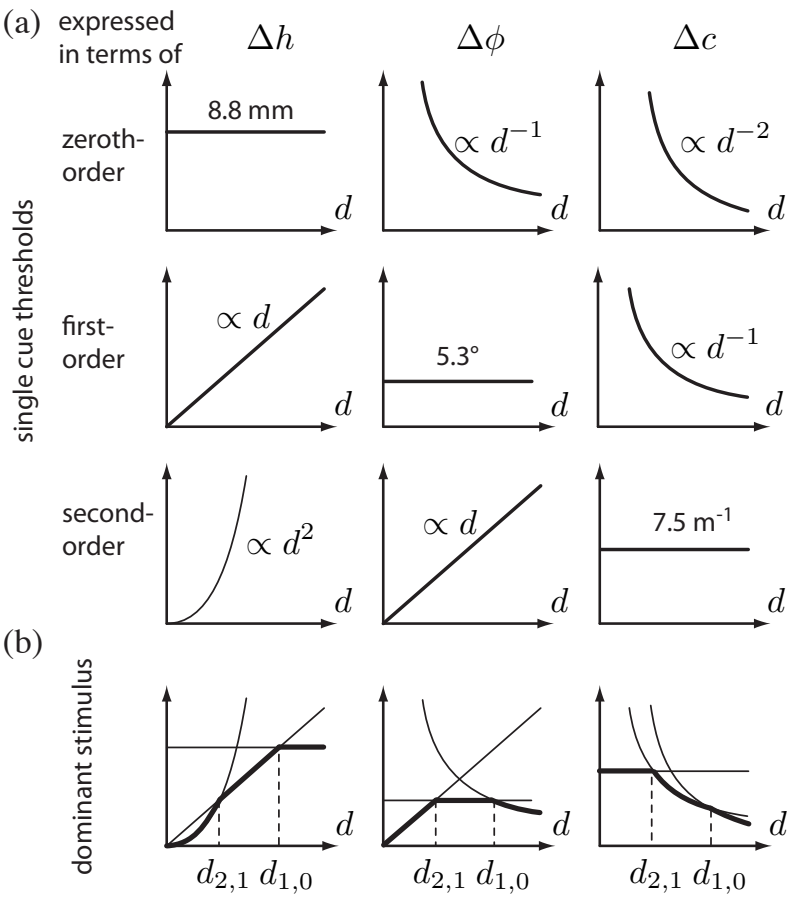

Fig. 8. (a) Trends of the change in isolated discrimination thresholds as a function of horizontal movement path length expressed with different quantities. (b) Cue dominance model obtained by combining individual sources of information. The scale space is separated in three ranges within which the different cues dominate.

The data of Pont et al. resulted from stimuli where all cues were combined. The data from Goodwin et al. specifically measures response to second-order information. With the apparatus used in Experiment 1, however, we could combine or segregate cues. By collecting all these results, we can build a more accurate picture of a haptic shape discrimination model by combining individual thresholds expressed with the same quantities.

Within the haptic modality, it is known that cues can combine statistically [6], or one can dominate the other [5]. However, the results of these studies cannot be directly applied to suggest a cue combination model since the cues were different and since the studies were performed at a fixed scale. On the other hand, it was shown that, at least in some regions of the stimulus space, first-order cues dominate completely as in Experiment 1 and reference [4]. To shed light on this issue, we proceeded in two steps. First, we estimated from our data and that from the literature the scales where the sources of geometrical information are operative, then we hypothesized cue integration models given the data collected so far.

1) Estimation of Ranges of Scales: Call $d_{1,0}$ the scale at which the discrimination thresholds for zeroth and first-order shape cues are equal. Below this value, a stimulus set at threshold in height would be above threshold in orientation. Above this value, a stimulus set at threshold in height would be below threshold in orientation. A transition value, $d_{2,1}$, can be similarly defined for orientation and curvature cues. These scale transition values are indicated in Fig. 8(b) where the thick lines show the ranges where an individual cue provides more accuracy than the other two. In particular, the intermediate range between $d_{2,1}$ and $d_{1,0}$ is the range where first-order geometrical cues are the most sensitive. The two points $d_{2,1}$ and $d_{1,0}$ can be calculated by using the two equations for $d$ from Table I. For $h, \phi$ and $c$, the threshold values from Table II can be used. Based on the results of Experiment 1 and of those of reference [11] we could estimate $d_{2,1}$ and $d_{1,0}$ to be $1.2 \mathrm{~cm}$ and $78 \mathrm{~cm}$, respectively. The orientation cue would dominate between fingertip size scales up to whole arm movements.

2) Possible Cue Integration Models: The simplest approach would be to posit that at any given scale one single cue dominates. The thick lines in Fig. 8(b) represent such a model expressed in terms of zeroth, first and second-order cues. To better understand the relationships between curvature thresholds for different exploration ranges, we plotted in Fig. 9 values from literature expressed in curvature values like in the rightmost panel of Fig. 8(b). Fig. 9 is plotted in log-log scale, so the power laws appear as straight line with slopes $0,-1$, and -2 . In this figure, the $84 \%$ threshold values for isolated cues are from Experiment 1 and from Goodwin et al. [11] (thick grey lines).

The same figure also plots other data from the literature grouped by 'full-cue' conditions (black symbols, Pont et al. [3], Van der Horst and Kappers [14], Gordon and Morison [2], condition (d)), and 'position-only-cue' conditions (grey symbols, Henriques and Soechting [7], condition (a), Drewing and Ernst [6]).

The similarity between the slope of the thresholds obtained from full-cue conditions and from the orientation-onlycue condition is quite striking (thin black line). The lower thresholds found with actual shapes can be explained by nongeometric cues used by humans when exploring real surfaces. Such non-geometrial cues include force fields arising from actual contacts since it is known that force fields can contribute as shape cues [5], [6], [15]. A very similar observation can be 


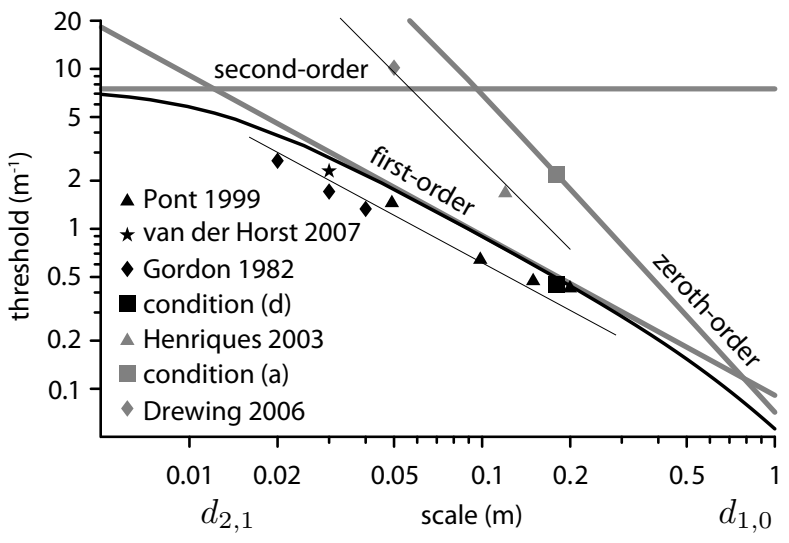

Fig. 9. The values from literature plotted in log-log scales. The grey data points indicate thresholds obtained from zeroth order information only. The black data points were obtained with real shapes. The grey lines indicate the model of lower right panel in Fig. 8 which appears as straight lines. The black curve is based on the hypothesis of weak fusion.

made of the thresholds found using stimuli containing only zeroth-order information which are also on a slope predicted by our model. The downward shift compared to the results of Experiments 1, condition (a), can be explained by variations in testing conditions. In our experiments we used a moving contact surface whereas in the studies described in references [7] and [6], subjects interacted with virtual shapes using a whole-hand manipulandum and a stylus, respectively. These methods of interaction might provide richer information than a single finger contact with a surface. Finally, it can be observed that stimuli giving only zeroth-order information yield considerably higher thresholds than real shapes or first-order cues conditions. Fig. 9 also indicates a model where cues combine statistically according to the weak fusion hypothesis [16], $\frac{1}{\sigma^{2}}=\sum \frac{1}{\sigma_{i}^{2}}$, (black curve). However, there is not sufficient data outside the range $\left[d_{2,1}, d_{0,1}\right]$ to support or strengthen this hypothesis.

Another source of information is the findings of Louw et al. [17]. They found that the increase of detection thresholds with increasing Gaussian stimulus width was described by a power law. The fitted coefficient was about 1.3 indicating that the relevant geometrical information is mostly first-order (coefficient $=1$ ) rather than second-order (coefficient $=2$ ). Our model also predicts that haptic shape discrimination depends only on the first and second-order cues, where the first-order cues dominates across a large range of scales.

3) The Relation Between Discrimination Performance and Haptic Perception of Objects: In this study we have examined the ability of subjects to discriminate a curved surface from a flat one, although the protocol used may lead to believe that it was a detection experiment. A standard detection experiment employs a single stimulus that must be distinguished from noise. Such experiment would not be appropriate due to confounding factors such as hysteresis and memory effects. Our results relate to shape perception in the sense that we found that first-order information is the dominant source of information through a large range of scale, which does not preclude the possibility that second-order information is also used. Nevertheless, our results show that differences in curvature are not well discriminated from second-order information.

\section{CONClusion}

In a first experiment, we measured the discrimination thresholds of human observers when exposed to shape stimuli that selectively delivered zeroth and first-order shape information, that is position and slope. We also tested the subjects in the condition where these cues were delivered together as well as with equivalent real objects. We found that for an exploration range of $18 \mathrm{~cm}$, shape discrimination depended mostly on first-order information. In a second experiment, we asked the question whether orientation-only shapes actually felt curved. A new group of participants was recruited to decide which of two stimuli felt more curved. One stimulus was real and the other contained either orientation-only information or orientation combined with position information. All participants showed biases that did not depend on the presence or absence of position information, demonstrating that in all cases they felt the stimuli as being curved.

The experiments were performed on a fixed exploration scale. We could nevertheless develop a model that applies to arbitrary scales. This model agrees with a number of results found in the literature which were measured using different exploration scales (see Fig. 9). Small scales are defined as stimuli shaped to provide curvature information within a distance commensurate with the size of a finger tip. Intermediate scale refers to a range spanning $1.2 \mathrm{~cm}$ to $78 \mathrm{~cm}$. Such a range of scales is accessible only through active touch. Subjects have no other option but explore the stimuli actively. Within this intermediate range, our model shows that the orientation-only-cue or first-order geometric information is dominant. Our model does not yet account for non-geometric cues which are known to be important for haptic shape perception. The presence or absence of these nongeometrical cues may explain the individual differences found in Experiment 2.

As far as the design of shape-simulating haptic interfaces is concerned, the conclusion is unequivocal. Perceptual performance is dramatically dependent on contact orientation information. This idea has already been successfully put in practice [18]-[20]. Not only does the addition of first-order information improve discriminability, but more importantly, evokes the subjective experience of feeling a curved surface as we have shown in Experiment 2 and in [4].

Our data suggest that haptic interfaces designed to deliver low curvature shape information might as well leave out the zeroth-order geometric information. The biases found in Experiment 2 are similar for shapes rendered with and without zeroth-order information. Also, the results of psychophysical experiments investigating haptic perception of shape where surface orientation is not available to the subjects should be discussed in the light of our results. For instance, studies such as that of Henriques and Soechting give insight into motor planning and control issues [7], but neglects to consider important factors governing the haptic perception of shape. 


\section{ACKNOWLEDGEMENTS}

The authors would like to thank Vincent Levesque for realtime programming assistance, Jerome Pasquero and Wouter Bergmann Tiest for insightful comments on the manuscript, and Hanifa Dostmohamed for the initial design and construction of the apparatus.

This research was supported by a grant from the Netherlands Organisation for Scientific Research (NWO) for Astrid Kappers, a grant from the Student Exchange Program of the IEEE Technical Committee on Haptics for Maarten Wijntjes, and a Discovery Grant from the Natural Sciences and Engineering Council of Canada (NSERC) for Vincent Hayward.

\section{REFERENCES}

[1] V. Hayward, "Haptic shape cues, invariants, priors, and interface design," in Human Haptic Perception - Basics and Applications, M. Grünwald, Ed. Birkhauser Verlag, 2008, pp. 381-392.

[2] I. Gordon and V. Morison, "The haptic perception of curvature," Perception and Psychophysics, vol. 31, pp. 446-450, 1982.

[3] S. C. Pont, A. M. L. Kappers, and J. J. Koenderink, "Similar mechanisms underlie curvature comparison by static and dynamic touch," Perception and Psychopysics, vol. 61, no. 5, pp. 874-894, 1999.

[4] H. Dostmohamed and V. Hayward, "Trajectory of contact region on the fingerpad gives the illusion of haptic shape," Experimental Brain Research, vol. 164, no. 3, pp. 387-394, 2005.

[5] G. Robles-De-La-Torre and V. Hayward, "Force can overcome object geometry in the perception of shape through active touch," Nature, vol. 412, pp. 445-448, 2001.

[6] K. Drewing and M. O. Ernst, "Integration of force and position cues for shape perception through active touch," Brain Research, vol. 1078, pp. 92-100, 2006

[7] D. Y. P. Henriques and J. F. Soechting, "Bias and sensitivity in the haptic perception of geometry," Experimental Brain Research, vol. 150, no. 1, pp. $95-108,2003$.

[8] A. E. Kirkpatrick and S. A. Douglas, "A shape recognition benchmark for evaluating usability of a haptic environment," in Proceedings of the First International Workshop on Haptic Human-Computer Interaction, S. Brewster and R. Murray-Smith, Eds. Berlin:Springer Verlag, 2002, pp. 151-156.

[9] V. Hayward, "Display of haptic shape at different scales," in Proceedings of Eurohaptics 2004, 2004, pp. 20-27.

[10] M. W. A. Wijntjes, A. Sato., A. K. L. Kappers, and V. Hayward, "Haptic perception of real and virtual curvature," in Haptics: Perception, Devices and Scenarios, ser. Lecture Notes in Computer Science, 2008, vol. 5024, pp. 361-366.

[11] A. W. Goodwin, K. T. John, and A. H. Marceglia, "Tactile discrimination of curvature by humans using only cutaneous information from the fingerpads," Experimental Brain Research, vol. 86, pp. 663-672, 1991.

[12] S. Coren, The left-hander syndrome. New York: Vintage Books, 1993.

[13] F. Wichmann and N. Hill, "The psychometric function i: Fitting, sampling and goodness of fit," Perception and Psychophysics, vol. 63, no. 8, pp. 1293-1313, 2001.

[14] B. J. van der Horst and A. M. L. Kappers, "Curvature discrimination in various finger conditions," Experimental Brain Research, vol. 177, no. 3, pp. 304-311, 2007.

[15] O. Portillo-Rodriguez, C. A. Avizzano, M. Bergamasco, and G. RoblesDe-La-Torre, "Haptic rendering of sharp objects using lateral forces," in Proceedings of the IEEE International Symposium on Robot and Human Interactive Communication (RO-MANO6), 2006, pp. 431-436.

[16] J. J. Clark and A. L. Yuille, Data fusion for sensory information processing systems. Boston, MA: Kluwer, 1990.

[17] S. Louw, A. M. L. Kappers, and J. J. Koenderink, "Haptic detection thresholds of gaussian profiles over the whole range of spatial scales," Experimental Brain Research, vol. 132, pp. 369-374, 2000.

[18] W. R. Provancher, M. K. Cutkosky, K. J. Kuchenbecker, and G. Niemeyer, "Contact location display for haptic perception of curvature and object motion," International Journal of Robotics Research, vol. 24, no. 9, pp. 1-11, 2005.

[19] Y. Yokokohji, N. Muramori, Y. Sato, and T. Yoshikawa, "Designing an encountered-type haptic display for multiple fingertip contacts based on the observation of human grasping behaviors," International Journal of Robotics Research, vol. 24, no. 9, pp. 717-729, 2005.
[20] M. Solazzi, A. Frisoli, F. Salsedo, and M. Bergamasco, "A fingertip haptic display for improving local perception of shape cues," in Proceedings of the Second Joint Eurohaptics Conference and Symposium on Haptic Interfaces for Virtual Environment and Teleoperator Systems, World Haptics 2007, 2007, pp. 409-414.

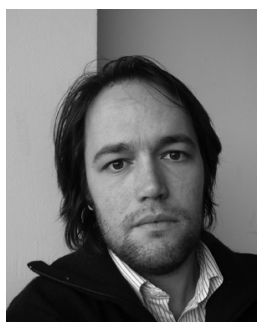

Maarten Wijntjes received his master degree in physics at the University of Groningen in 2003. In 2004 he started his $\mathrm{PhD}$ project at the Physics of Man group of the Utrecht University. During that time he worked on haptic perception of raised line drawings, haptic mental rotation and haptic perception of shape. In 2008 and he received his $\mathrm{PhD}$ degree and started working as a post-doc for dr. Sylvia Pont at Delft University of Technology on a project entitled 'ecological optics'.

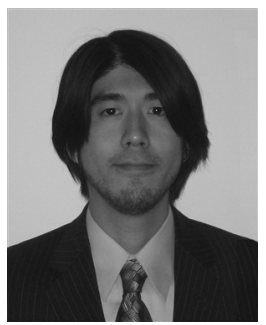

Akihiro Sato received the BSc degree in mechanical engineering from Aoyama Gakuin University and the MEng degree in mechanical engineering from McGill University. He also has experience as an engineer in the real-time hardware-in-the-loop simulation industry. He is currently a research engineer at the Centre for Intelligent Machines of McGill University. His research interests include sensory-motor control of humans and animals, dynamic motion control of robotic machines, and geometric control theory applied to the aforementioned systems.

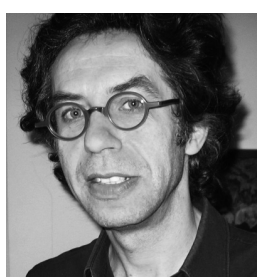

Vincent Hayward (M'84-SM'04-FIEEE'08), Ing. Ecole Centrale de Nantes 1978; Ph.D. Computer Science 1981, University of Paris; Visiting Assistant Professor, Purdue University (1982); Charg é de Recherches at CNRS, France (1983-86), Professeur invité, Université Pierre et Marie Curie, UPMC (2006); is Professor of Electrical and Computer Engineering at McGill University and has recently taken up a chaired position at UPMC. Hayward is interested in haptic device design and applications, perception, and robotics. He is leading the Haptics Laboratory at McGill University since 1994 and was the Director of the Center for Intelligent Machines (2001-2004). He is a co-Founder of the Experimental Robotics Symposia, Program Vice-Chair 1998 IEEE Conference on Robotics and Automation, Program Vice-Chair ISR2000, past Associate Editor of the IEEE Transactions on Robotics and Automation, now member of the Governing board of Haptics-e, of the Editorial board of the ACM Transactions on Applied Perception, and of the IEEE Transactions on Haptics. Hayward received best paper and research awards including the NASA Space Act Tech Brief Award (1991) and the E. (Ben) \& Mary Hochhausen Award for Research in Adaptive Technology For Blind and Visually Impaired Persons (2002).

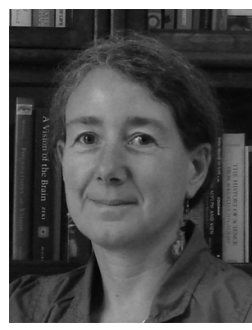

Astrid Kappers studied experimental physics at Utrecht University, the Netherlands. She got her PhD from Eindhoven University of Technology. Since 1989 she works in the department of Physics and Astronomy of Utrecht University. She was promoted to full professor in 2005. Her research takes place in the Helmholtz Institute. Her research interests include haptic and visual perception. In 2003 she won the prestigious VICI grant. She is member of the editorial boards of Acta Psychologica and Current Psychology Letters and associate editor of IEEE Transactions on Haptics. 